\title{
PATRONES DE PROCESAMIENTO SENSORIAL E INTERVENCIÓN TEMPRANA EN NIÑOS MENORES DE 3 AÑOS: UNA REVISIÓN DE LA LITERATURA
}

\author{
SENSORY PROCESSING PATTERNS AND EARLY INTERVENTION IN CHILDREN \\ UNDER 3 YEARS OF AGE: A REVIEW OF THE LITERATURE
}

\section{Juliana Gutiérrez Faccini ${ }^{1}$}

\begin{abstract}
RESUMEN
La presente revisión se centra en proporcionar una visión general de la literatura que identifica estudios enfocados en definir los patrones de procesamiento sensorial en niños menores de 3 años y los enfoques de intervención identificados en esta población. Durante la última década ha existido un crecimiento constante de la literatura relacionada con el procesamiento sensorial en los niños pequeños, sin embargo, la información disponible entre las diferentes publicaciones es aún limitada y segmentada. En esta revisión se recopilaron 15 artículos sobre los patrones de procesamiento sensorial en los niños pequeños y 10 artículos relacionados con los enfoques de intervención para un total de 25 artículos publicados entre 2007 y 2017. Del análisis de la información obtenida acerca de los patrones de procesamiento sensorial, surgieron los siguientes temas: el diagnóstico y los factores de riesgo, el tipo de disfunción del procesamiento sensorial y el impacto funcional. Entre los temas que surgieron al analizar los enfoques de intervención, se destaca la importancia de la familia como un tema general que abarca subtemas de intervención, tales como: estrategias sensoriales, capacitación de los padres e intervenciones grupales. Si bien es necesario continuar desarrollando investigaciones que amplíen nuestro conocimiento en relación con estos temas en general, la evidencia apoya la efectividad de la intervención temprana.
\end{abstract}

\section{PALABRAS CLAVES}

Desarrollo infantil, terapia ocupacional, procesamiento sensorial, disfunción sensorial, intervención temprana.

\begin{abstract}
The present review has focused on providing a general view of the literature related to the identification of studies that define the sensory processing patterns of children under the age of 3 years as well as recognizing the intervention approaches identified in this population. Although there seems to be a consistent grow of literature over the last 10 years related to the sensory processing patterns of young children, the amount of research is still limited and compartmentalized. A total of 15 articles were collected regarding the sensory processing patterns of young children and 10 articles related to the intervention approaches, for a total of 25 articles published between 2007 and 2017 . Several themes emerged in the analysis of the information for the sensory processing patterns that included the diagnosis and risk factors, the type of sensory processing dysfunction, and the functional impact. Themes also emerged when analyzing the intervention approaches, highlighting the importance of the family as an overarching theme and related subthemes such as: sensory strategies, parent training, and group interventions. Although there is a need for future research in these areas overall, there is positive evidence supporting the effectiveness of early intervention.
\end{abstract}

\section{KEYWORDS}

Child development, treatment outcomes, sensory processing, sensory disorders, occupational therapy.

1 Terapeuta Ocupacional de la Escuela Colombiana de Rehabilitación adjunta a la Universidad de Nuestra Señora del Rosario. Master en Terapia Ocupacional de la Universidad del Sur de California. Doctorado Clínico de la Universidad del Sur de California 6345 Green Valley Cr. Culver City, California 90230 Julianaotr@hotmail.com.Número ORCID: 0000-0003-2294-1365 


\section{INTRODUCCIÓN}

El procesamiento sensorial juega un rol significativo en el desarrollo de los niños que comienza antes de su nacimiento. En la medida que los niños se desarroIlan están constantemente organizando sensaciones para desarrollar habilidades motoras, regularse emocionalmente, aprender nuevas habilidades, enfocar su atención, y relacionarse con el ambiente y con otros (Ayres, 2005).

Durante la última década se han hecho avances en el estudio y la descripción de disfunciones sensoriales en distintos diagnósticos, sin embargo, la información esta segmentada entre las diferentes publicaciones disponibles. Existen pocas revisiones de la literatura en este tema que ayuden a organizar la información. Una de ellas es la de Blanche y Gunter (2020), que se basa en la literatura existente para dividir los desórdenes en procesamiento sensorial en identificación temprana en dos tipos: desordenes de la regulación y desordenes sensoriomotores. De acuerdo con la literatura, los desórdenes de la regulación son de tres tipos: hiper-respuesta, hipo-respuesta y otros desordenes de regulación que incluyen fluctuaciones, búsqueda de sensaciones y conductas repetitivas. Los desórdenes de regulación afectan la atención, las respuestas emocionales, la alimentación y las conductas adaptativas. Los desórdenes sensoriomotores son menos estudiados y afectan planeamiento motor, imitación y control postural. Estos desórdenes incluyen desordenes perceptivo-motores y desordenes de coordinación motora gruesa (Blanche y Gunter, 2020).

La literatura ha destacado a través de los años la importancia de las primeras experiencias del niño en el desarrollo del cerebro (DeMaster, et al., 2019; Koch y Moore, 2015; Mundkur, 2005), por esta razón, en la intervención temprana se buscan experiencias positivas y dinámicas con el medio ambiente social y físico que contribuyan a fortalecer la participación del niño dentro de su entorno, y a desarrollar habilidades en varias áreas (motor, socio-emocional, cognitivo, comunicativo, y de autocuidado) (Clark y Kinsgley, 2020; Park, Maitra, Achon, Loyola y Rincón, 2014). La información sensorial forma parte de estas experiencias, puede influenciar las respuestas del niño y producir cambios en su comportamiento. Una revisión de la literatura de Lane y Schaaf (2010) estableció la relación entre información sensorial, función cerebral y comportamiento. Adicionalmente, la Asociación Americana de Terapia Ocupacional (AOTA) realizó una serie de revisiones sistemáticas que destaca la evidencia de la intervención temprana (Arbesman, Lieberman, y Berlanstein, 2013) en varias áreas: desarroIlo socio-emocional (Case-Smith, 2013), alimentación (Howe y Wang, 2013), desarrollo cognitivo (Frolek Clark y Schlabach, 2013), desarrollo motor (Case-Smith, Frolek Clark, y Schlabach, 2013), y modelos de entrega de los servicios (Kingsley y Mailloux, 2013).

El propósito de esta revisión es proporcionar una visión general de la literatura con relación a: 1. Los patrones en el procesamiento sensorial identificados en los niños menores de 3 años, y 2. Los enfoques de intervención identificados en este grupo de edad.

\section{MÉTODO}

El presente estudio realiza una revisión exploratoria general ("scoping study") de tipo descriptiva, como método de búsqueda para identificar la literatura relevante. La revisión fue guiada por las siguientes preguntas: "Qué se conoce en la literatura clínica y empírica existente con relación al procesamiento sensorial en niños menores de 3 años?" y "qué tipo de intervenciones se han documentado a esta temprana edad?". Una búsqueda inicial identificó artículos utilizando palabras claves en los títulos y en el resumen de los artículos. La búsqueda se limitó a artículos publicados en inglés en un período de 10 años, desde 2007 hasta 2017 (momento de esta exploración). Las palabras claves de búsqueda evolucionaron, guiados por los siguientes términos: sensorial, problemas sensoriales, integración sensorial, procesamiento sensorial, intervención, intervención temprana, niños, estrategias sensoriales, pediatría, modulación, regulación, o una combinación de éstos. En la búsqueda se utilizaron varias bases de datos tales como Medline, OVID, CINAHL, Google Scholar, PubMed, y COCHRANE. Aquellos artículos 
cuyos participantes incluían un rango de edad muy amplio, específicamente niños mayores de 6 años o que contemplaban una discusión anatomo-fisiológica de los sistemas sensoriales fueron excluidos. Se incluyeron artículos de literatura cualitativa o cuantitativa que explícitamente discutían características sensoriales o disfunción sensorial en niños menores de 3 años (independiente del diagnóstico), o que mencionaban formas de intervención temprana no necesariamente relacionadas con aspectos sensoriales. Por último, se hizo la revisión y el análisis del texto completo de los artículos, clasificándolos por temas comunes y proporcionando un resumen en forma tabular para organizar la información existente.

\section{Resultados}

De un total de 69 artículos analizados en la búsqueda inicial, se seleccionaron 15 que analizaban los patrones sensoriales y 10 que incluían enfoques de intervención en la población menor de 3 años. A continuación, se presentan dos líneas de análisis de la información: Patrones de procesamiento sensorial y enfoques de intervención identificados para esta población. Cada sección discute los temas que emergieron durante el análisis.

\section{Patrones de procesamiento sensorial}

Los temas que surgieron relacionados con los patrones de procesamiento sensorial se agruparon de la siguiente forma: (a) diagnóstico y factores de riesgo; (b) tipos de disfunción del procesamiento sensorial, y (c) impacto funcional que tienen estos déficits en los niños (Tabla 1).

TABLA 1. ARTíCULOS RELACIONADOS CON LOS PATRONES DE PROCESAMIENTO SENSORIAL.

\begin{tabular}{|c|c|c|c|}
\hline $\begin{array}{l}\text { Temas } \\
\stackrel{\rightarrow}{\text { Autores }}\end{array}$ & $\begin{array}{l}\text { Dx / Factores } \\
\text { de Riesgo }\end{array}$ & $\begin{array}{c}\text { Disfunción del Procesamiento } \\
\text { Sensorial }\end{array}$ & Impacto Funcional \\
\hline $\begin{array}{l}\text { Baranek } \\
\text { et al., } \\
2007\end{array}$ & $\begin{array}{l}\text { TEA, RD, } \\
\text { DT }\end{array}$ & $\begin{array}{l}\text { Hyper-respuesta es > en TEA y RD que en DT. } \\
\text { Disminuye con edad mental y cronológica entre } \\
\text { los grupos. TEA menos probable que responda } \\
\text { al timbre del teléfono a los } 6 \text { m. No hay diferen- } \\
\text { cia a los } 5.5 \text { años en relación a RD y DT. }\end{array}$ & \\
\hline $\begin{array}{l}\text { Baranek } \\
\text { et al. } \\
2013\end{array}$ & TEA & $\begin{array}{l}\text { A los } 6 \mathrm{~m} \text {. la hipo-respuesta es más significativa } \\
\text { en niños con TEA que RD y DT (> a estímulos } \\
\text { sociales que no sociales). } \\
\text { No significativo a los } 60 \mathrm{~m} \text {. }\end{array}$ & $\begin{array}{l}\text { La hipo-respuesta a los estí- } \\
\text { mulos sociales y no sociales } \\
\text { predice niveles más bajos de } \\
\text { atención conjunta y lenguaje } \\
\text { en niños con TEA. }\end{array}$ \\
\hline
\end{tabular}




\begin{tabular}{|c|c|c|c|}
\hline $\begin{array}{c}\text { Temas } \\
\rightarrow \\
\text { Autores }\end{array}$ & $\begin{array}{c}\text { Dx / Factores } \\
\text { de Riesgo }\end{array}$ & $\begin{array}{c}\text { Disfunción del Procesamiento } \\
\text { Sensorial }\end{array}$ & Impacto Funcional \\
\hline $\begin{array}{l}\text { Ben-Sas- } \\
\text { son et al. } \\
2007\end{array}$ & TEA & $\begin{array}{l}\text { Hiper-respuesta e hipo-respuesta extremas son } \\
\text { significativamente más comunes en el grupo de } \\
\text { TEA. } \\
\text { El grupo TEA tuvo puntajes significativamente } \\
\text { más altos en al menos } 2 \text { cuadrantes para 'regis- } \\
\text { tro bajo', 'sensibilidad' y 'evitación' del perfil } \\
\text { sensorial frente a DT. El grupo TEA tuvo puntajes } \\
\text { significativamente más bajos para la 'búsqueda' } \\
\text { que el grupo de la misma edad mental pero no } \\
\text { con el grupo de la misma edad cronológica. }\end{array}$ & \\
\hline $\begin{array}{l}\text { Bröring } \\
\text { et al. } \\
2017\end{array}$ & $\begin{array}{l}\text { UCIN / } \\
\text { niño } \\
\text { pre-término }\end{array}$ & $\begin{array}{l}\text { Los recién nacidos prematuros mostraron signi- } \\
\text { ficativamente más problemas en la modulación } \\
\text { sensorial (Ej., Auditiva, táctil, vestibular). } \\
\text { La naturaleza y la gravedad diferían ampliamen- } \\
\text { te entre los estudios, posiblemente debido a la } \\
\text { variabilidad de las medidas y los tipos de pro- } \\
\text { blemas de los niños prematuros. }\end{array}$ & $\begin{array}{l}\text { Todos los estudios mostra- } \\
\text { ron que los recién nacidos } \\
\text { prematuros tuvieron un peor } \\
\text { desempeño funcional. } \\
\text { Hubo una variedad de fun- } \\
\text { ciones (integración visual- } \\
\text { táctil, motor adaptativo), de } \\
\text { patrones de comportamiento } \\
\text { resultantes (ej, bajo registro, } \\
\text { búsqueda de sensaciones) y } \\
\text { de gravedad del impacto. } \\
\text { Solo } 2 \text { de } 5 \text { estudios encon- } \\
\text { traron relación entre proble- } \\
\text { mas de modulación y desa- } \\
\text { rrollo cognitivo. }\end{array}$ \\
\hline $\begin{array}{l}\text { Chorna } \\
\text { et al., } \\
2014\end{array}$ & Prematuro & $\begin{array}{l}\text { El } 82 \% \text { de los lactantes tenían reactividad sen- } \\
\text { sorial en al menos } 1 \text { de } 5 \text { categorías, siendo las } \\
\text { más afectadas los estímulos de presión profun- } \\
\text { da ( } 49 \%) \text { y vestibulares ( } 21 \%) \text {. } \\
\text { La modulación sensorial aumentó a medida } \\
\text { que disminuyó la edad gestacional. }\end{array}$ & $\begin{array}{l}\text { El } 73 \% \text { completó la prueba } \\
\text { de Bayley, presentando pun- } \\
\text { tajes más bajos en las escalas } \\
\text { motora y de lenguaje. Los } \\
\text { puntajes deficientes en el } \\
\text { control motor ocular se aso- } \\
\text { ciaron con puntajes bajos a } \\
\text { nivel cognitivo y motores en } \\
\text { la infancia. }\end{array}$ \\
\hline
\end{tabular}




\begin{tabular}{|c|c|c|c|}
\hline $\begin{array}{c}\text { Temas } \\
\rightarrow \\
\text { Autores }\end{array}$ & $\begin{array}{c}\text { Dx / Factores } \\
\text { de Riesgo }\end{array}$ & $\begin{array}{c}\text { Disfunción del Procesamiento } \\
\text { Sensorial }\end{array}$ & Impacto Funcional \\
\hline $\begin{array}{l}\text { DeSan- } \\
\text { tis et al. } \\
2011\end{array}$ & $\begin{array}{l}\text { Infantes a } \\
\text { término }\end{array}$ & $\begin{array}{l}\text { Hubo una fuerte relación entre la disfunción del } \\
\text { procesamiento sensorial (reflejada en una tole- } \\
\text { rancia reducida a los estímulos sensoriales en el } \\
\text { perfil sensorial de bebés) con el temperamento } \\
\text { (calificados como niños más difíciles, con mayor } \\
\text { problema para calmarlos, y más activos). }\end{array}$ & $\begin{array}{l}\text { Existe una relación entre la } \\
\text { capacidad del bebé para per- } \\
\text { manecer regulado/alerta y su } \\
\text { grado de competencia moto- } \\
\text { ra. A mayor competencia } \\
\text { en autorregulación y menor } \\
\text { nivel de alerta, mayor calidad } \\
\text { y madurez del movimiento } \\
\text { y los reflejos, mayor estado } \\
\text { alerta al orientarse a los estí- } \\
\text { mulos y menor respuestas } \\
\text { autonómicas de estrés. }\end{array}$ \\
\hline $\begin{array}{l}\text { Germani } \\
\text { et al, } \\
2014\end{array}$ & $\begin{array}{l}\text { TEA } \\
\text { (alto y bajo } \\
\text { riesgo) } \\
\text { ITSP }\end{array}$ & $\begin{array}{l}\text { A los } 24 \mathrm{~m} \text {. diferencia significativa en el proce- } \\
\text { samiento auditivo para TEA. No se detectaron } \\
\text { diferencias en secciones visuales, vestibulares, } \\
\text { táctiles ni orales. TEA en riesgo también exhibió } \\
\text { a los } 24 \mathrm{~m} \text {. una diferencia significativa para el } \\
\text { registro bajo. Alto riesgo-N y bajo riesgo dentro } \\
\text { del rango típico. } \\
\text { No hay diferencias en 'búsqueda' entre los } \\
\text { grupos. }\end{array}$ & \\
\hline $\begin{array}{l}\text { Gutie- } \\
\text { rrez et al. } \\
2016\end{array}$ & $\begin{array}{l}\text { TEA, RD, } \\
\text { DT }\end{array}$ & $\begin{array}{l}\text { Diferencia significativa entre niños con TEA y } \\
\text { RD en 'bajo registro'. No hay diferencia signifi- } \\
\text { cativa en 'búsqueda de sensaciones' entre los } \\
\text { tres grupos. Diferencia significativa entre niños } \\
\text { con TEA y RD en el procesamiento auditivo y } \\
\text { sensorial oral. }\end{array}$ & \\
\hline $\begin{array}{l}\text { Mitchell } \\
\text { et al., } \\
2015\end{array}$ & Prematuro & $\begin{array}{l}\text { La mayoría de los artículos se relacionan con } \\
\text { trastorno de modulación sensorial de tipo } \\
\text { hiper-respuesta (principalmente táctiles y visua- } \\
\text { les). Pocos artículos fueron relacionados con } \\
\text { hipo-respuesta y menos con SBMD. }\end{array}$ & \\
\hline
\end{tabular}




\begin{tabular}{|c|c|c|c|}
\hline $\begin{array}{l}\text { Temas } \\
\stackrel{\rightarrow}{\text { Autores }}\end{array}$ & $\begin{array}{c}\text { Dx / Factores } \\
\text { de Riesgo }\end{array}$ & $\begin{array}{c}\text { Disfunción del Procesamiento } \\
\text { Sensorial }\end{array}$ & Impacto Funcional \\
\hline $\begin{array}{l}\text { Mulligan } \\
\text { \& White, } \\
2012\end{array}$ & $\begin{array}{l}\text { Infante de } \\
\text { alto riesgo } \\
\text { de TEA }\end{array}$ & $\begin{array}{l}\text { Grupos de alto riesgo tuvo puntuaciones de } \\
\text { procesamiento sensorial que reflejaron menos } \\
\text { conductas de 'búsqueda sensorial' (significa- } \\
\text { tivas) y más dificultades en el procesamiento } \\
\text { auditivo (no significativa) que el grupo de bajo } \\
\text { riesgo. }\end{array}$ & $\begin{array}{l}\text { No hay diferencias en las formas } \\
\text { de juego funcional, sensorio- } \\
\text { motora y asociativa. No hay } \\
\text { diferencias en comportamientos } \\
\text { sensoriales o motores durante la } \\
\text { alimentación. } \\
\text { A pesar de los puntajes típicos en } \\
\text { el desarrollo en el grupo de alto } \\
\text { riesgo, la calidad de los movi- } \\
\text { mientos motores gruesos y finos } \\
\text { carecía de refinamiento durante } \\
\text { el juego libre. }\end{array}$ \\
\hline $\begin{array}{l}\text { Philpott- } \\
\text { Robinson } \\
\text { et al, } \\
2016\end{array}$ & TEA & $\begin{array}{l}\text { Las dificultades de procesamiento sensorial oral } \\
\text { fueron moderadamente correlacionadas, pero } \\
\text { significativamente negativa con la puntuación } \\
\text { ADEC. }\end{array}$ & \\
\hline $\begin{array}{l}\text { Smith et } \\
\text { al. } 2014\end{array}$ & $\begin{array}{l}\text { Alto y bajo } \\
\text { riesgo para } \\
\text { TEA }\end{array}$ & $\begin{array}{l}\text { Grupo de alto riesgo diagnosticado con TEA } \\
\text { después de } 3 \text { años tuvo significativamente } \\
\text { menos registro sensorial a los } 24 \text { meses que los } \\
\text { grupos de bajo o alto riesgo no diagnosticados } \\
\text { con TEA a los } 3 \text { años. Grupo de alto riesgo diag- } \\
\text { nosticado con TEA tuvo significativamente más } \\
\text { dificultades en el procesamiento auditivo. } \\
\text { No se detectaron diferencias para los dominios } \\
\text { de procesamiento sensorial visual, vestibular, } \\
\text { táctil u oral. Tendencia no significativa hacia las } \\
\text { diferencias en el cuadrante de búsqueda para el } \\
\text { grupo alto riesgo diagnosticado con TEA versus } \\
\text { otros dos grupos. }\end{array}$ & \\
\hline $\begin{array}{l}\text { Tauman, } \\
\text { et al. } \\
2017\end{array}$ & $\begin{array}{l}\text { Alteración } \\
\text { en alimenta- } \\
\text { ción y sueño. }\end{array}$ & $\begin{array}{l}\text { Después de controlar por edad, el grupo de } \\
\text { trastornos de la alimentación mostró una dife- } \\
\text { rencia significativa en el ITSP en comparación } \\
\text { con el grupo control en "procesamiento oral", } \\
\text { con mayores probabilidades de tener patro- } \\
\text { nes de sensibilidad sensorial, evitación de la } \\
\text { sensación y bajo umbral sensorial que el grupo } \\
\text { control. } \\
\text { El grupo de insomnio conductual mostró una } \\
\text { diferencia significativa en comparación con los } \\
\text { grupos de control y trastorno de alimentación } \\
\text { en el procesamiento oral con mayores probabi- } \\
\text { lidades de tener patrones de evitación de sen- } \\
\text { sación y umbral sensorial bajo que el grupo de } \\
\text { trastornos de alimentación o de control. }\end{array}$ & $\begin{array}{l}\text { La tendencia a la hiper res- } \\
\text { puesta en ambos grupos pue- } \\
\text { de explicar la coexistencia de } \\
\text { ambos problemas. }\end{array}$ \\
\hline
\end{tabular}




\begin{tabular}{|l|l|l|l|}
\hline $\begin{array}{c}\text { Temas } \\
\text { Autores }\end{array}$ & $\begin{array}{l}\text { Dx /Factores } \\
\text { de Riesgo }\end{array}$ & \multicolumn{1}{|c|}{$\begin{array}{c}\text { Disfunción del Procesamiento } \\
\text { Sensorial }\end{array}$} & \multicolumn{1}{c|}{ Impacto Funcional } \\
\hline $\begin{array}{l}\text { Vasak, et } \\
\text { al. 2015 }\end{array}$ & $\begin{array}{l}\text { Infantes con } \\
\text { alteraciones } \\
\text { de sueño }\end{array}$ & $\begin{array}{l}\text { Los niños con problemas de sueño tienden a } \\
\text { presentar una mayor sensibilidad en el ITSP } \\
(36 \%)\end{array}$ & $\begin{array}{l}\text { Una mayor sensibilidad se } \\
\text { relaciona con más tiempo } \\
\text { para conciliar el sueño. Los } \\
\text { niños que se ubican dentro } \\
\text { del patrón de búsqueda sen- } \\
\text { sorial duermen significativa- } \\
\text { mente menos durante el día } \\
\text { que DT }\end{array}$ \\
\hline $\begin{array}{l}\text { Weiss \& } \\
\text { Wilson, } \\
2006\end{array}$ & $\begin{array}{l}\text { UCIN } \\
\text { Infantes de } \\
\text { alto riesgo }\end{array}$ & $\begin{array}{l}\text { El número de procedimientos médicos peligro- } \\
\text { sos posnatales tuvo un 28\% de variación en la } \\
\text { probabilidad de desarrollar aumento en el nivel } \\
\text { de alerta e intranquilidad al contacto durante } \\
\text { los cuidados de atención. }\end{array}$ & \\
\hline
\end{tabular}

Nota: La Tabla 1 presenta los artículos que identifican los patrones de disfunción sensorial en los niños menores de 3 años. Nomenclaturas en el cuadro: DX = Diagnóstico; TEA = Trastorno del Espectro Autista; RD = niños con retraso en el desarrollo; DT = niños con desarrollo típico; ITSP = Infant Toddler Sensory Profile (perfil sensorial en el infante y niño pequeño); SBMD = Alteraciones motoras de base sensorial; $y$ ADEC = Instrumento de detección de autismo en la primera infancia.

En relación al diagnóstico y los factores de riesgo, hubo un claro interés de muchos investigadores en niños con trastorno del espectro autista (TEA) (Baranek, Boyd, Poe, David y Watson, 2007; Baranek, et al., 2013; Ben-Sasson, et al., 2007; Germani, et al., 2014; Gutiérrez, Chang y Blanche, 2016; Mulligan y White, 2012; Philpott-Robinson, Lane, y Harpster, 2016; Smith, et al., 2014) y prematuros o de alto riesgo, (Bröring, Oostrom, Lafeber, Jansma y Oosterkaan, 2017; Chorna, Solomon, Slaughter, Stark y Maitre, 2014; Mitchell, Moore, Roberts, Hachtel y Brown, 2015; Weiss y Wilson, 2006).

Las investigaciones revelan que las dificultades de procesamiento sensorial están presentes con mayor frecuencia en niños con TEA que en niños con retrasos en el desarrollo (RD) o niños con desarrollo típico (DT) (Baranek et al., 2007; Baranek et al., 2013; Ben-Sasson, et al., 2007). La mayoría de los autores identificaron que los niños con TEA tienden a presentar hipo-respuesta al estímulo sensorial en comparación con los niños con RD o DT (Baranek et al., 2007; Baranek et al., 2013; Germani et al., 2014; Gutiérrez, et al., 2016; Smith, et al., 2014), siendo más significativa para los estímulos sociales que para los no sociales (Baranek, et al., 2013). Otras disfunciones del procesamiento sensorial en el niño con TEA incluyeron déficits en el procesamiento auditivo de hiper-respuesta (Germani et al., 2014; Gutiérrez, et al., 2016; Mulligan y White, 2012; Smith, et al., 2014) y dificultades sensoriales orales (Gutiérrez, et al., 2016; Philpott-Robinson, et al., 2016), este último considerado por algunos autores como un signo temprano de tea. Dentro de los estudios analizados no se encontró ninguna diferencia estadísticamente significativa en los comportamientos de búsqueda sensorial entre los tres grupos, TEA, RD, y DT (Ben-Sasson et al., 2007; Germani et al., 2014; Gutiérrez et al., 2016; Mulligan y White, 2012; Smith, et al. 2014).

En contraste con las características de procesamiento sensoriales del niño con TEA, la mayoría de los estudios reportaron hiper-respuesta al estímulo sensorial en los recién nacidos prematuros o de alto riesgo. Se estima que esta hiper-respuesta se encuentra en un $82 \%$ de estos niños, afectando principalmente los sistemas auditivo y táctil, pero también se ha reportado en el sistema vestibular (Bröring et al., 2017; Chorna, et al., 2014; 
Mitchell et al., 2015; Weiss y Wilson, 2006). En la UCIN, varios factores de riesgo predijeron una mayor hiperrespuesta a la información sensorial, incluyendo: menor edad gestacional, bajo peso al nacer, mayor número de procedimientos médicos y exposición a medicamentos (Weiss y Wilson, 2006).

Al analizar el impacto funcional que tienen las disfunciones en el procesamiento sensorial en el niño con TEA, Baranek et al. (2013) encontraron que la hipo-respuesta a la información sensorial se asociaba con déficits en la atención conjunta y el lenguaje. Los problemas sensoriales relacionados con la hiper-respuesta en el niño prematuro se correlacionaron con déficits cognitivos (Bröring, et al., 2017) y puntajes bajos en las escalas de desarrollo motor y del lenguaje en la prueba Bayley 3Scales of Infant and Toddler Development (Chorna, et al., 2014). Otras áreas afectadas por la hiper-respuesta a la información sensorial incluyeron los trastornos de la alimentación y del sueño (Tauman, et al. 2017; Vasak, Williamson, Garden y Zwicker, 2015), las alteraciones en el temperamento del niño (DeSantis, Harkins, Tronick y Beeghly, 2011) y los niveles de alerta alto e intranquilidad al contacto durante las actividades de cuidado diario, como al bañarlos (Weiss y Wilson, 2006) (Tabla 2).

TABLA 2. RESUMEN DE LOS PATRONES DE DISFUNCIÓN SENSORIAL EN MENORES

\begin{tabular}{|l|l|l|}
\hline Población & Patrón de disfunción sensorial & Impacto funcional \\
\hline TEA & $\begin{array}{l}\text { Hipo-respuesta / Bajo registro } \\
\text { Hiper-respuesta auditiva } \\
\text { No hubo diferencia en comporta- } \\
\text { miento de búsqueda sensorial } \\
\text { Alteración sensorial oral }\end{array}$ & $\begin{array}{l}\text { Atención conjunta } \\
\text { Lenguaje } \\
\text { Alimentación }\end{array}$ \\
\hline $\begin{array}{l}\text { Prematuros o de alto } \\
\text { riesgo }\end{array}$ & $\begin{array}{l}\text { Hiper-respuesta (82\%) } \\
\text { > Tactil y auditiva } \\
\text { vestibular }\end{array}$ & $\begin{array}{l}\text { Desarrollo cognitivo } \\
\text { Bajos puntajes en escalas motora y } \\
\text { lenguaje del Bayley 3 } \\
\text { Sueño y temperamento } \\
\text { Alimentación } \\
\text { Cuidado diario (ej. bañarlos) }\end{array}$ \\
\hline
\end{tabular}

Nota: La Tabla 2 presenta los el resumen de los patrones de disfunción identificados en los artículos por población. Nomenclatura en el cuadro: TEA = Trastorno del Espectro Autista.

\section{Enfoques de intervención temprana}

Al analizar las prácticas de intervención temprana identificadas en la revisión, se destaca la importancia de la familia (Bulkeley, Bundy, Roberts y Einfeld, 2016; Colyvas, Sawyer y Campbell, 2010; Kingsley y Mailloux, 2013; Wainer, Hepburn y McMahon Griffith, 2017) dentro de la cual se contemplan tres subtemas: el uso de estrategias sensoriales, la educación de los padres, y las intervenciones grupales. Estos subtemas interactúan entre sí. Por ejemplo, los programas grupales enriquecidos sensorialmente corresponden tanto al subtema estrategias sensoriales como al subtema de intervenciones grupales, y de manera similar, la educación de los padres puede ocurrir durante las intervenciones relacionadas con la implementación de estrategias sensoriales o durante las intervenciones grupales (Tabla 3). 
TABLA 3 RESUMEN DE LAS INTERVENCIONES EN LA POBLACIÓN DE NIÑOS MENORES.

\begin{tabular}{|c|c|c|c|c|}
\hline $\begin{array}{l}\text { Temas } \\
\stackrel{\rightarrow}{\text { Autores }}\end{array}$ & Studio & Sensorial & Enfocado en el padre//familia & Grupal \\
\hline $\begin{array}{l}\text { Benzies et } \\
\text { al., } 2013\end{array}$ & $\begin{array}{l}\text { Revisión de } \\
18 \text { estudios } \\
\text { (prematuros). }\end{array}$ & & $\begin{array}{l}\text { Efectos positivos para la ansiedad, } \\
\text { la depresión y la autoeficacia de } \\
\text { las madres con efectos positivos } \\
\text { sobre el desarrollo del niño a } \\
\text { corto plazo hasta los } 24 \mathrm{~m} \text {. al } \\
\text { proporcionar retroalimentación } \\
\text { profesional y apoyo terapéutico } \\
\text { para el desarrollo (educación } \\
\text { para padres). }\end{array}$ & \\
\hline $\begin{array}{l}\text { Blanche } \\
\text { et al., } \\
2016\end{array}$ & $\begin{array}{l}\text { Intervención } \\
\text { Grupal } \\
\text { 18-36m }\end{array}$ & $\begin{array}{l}\text { Estrategias de base sen- } \\
\text { sorial (ej. ajustes para } \\
\text { sentarse, descansos } \\
\text { sensoriales/ } \\
\text { movimiento) y activida- } \\
\text { des sensoriales basa- } \\
\text { das en los principios } \\
\text { de la IS. Los resultados } \\
\text { muestran mejoría en } \\
\text { cognición y lenguaje (y } \\
\text { motricidad gruesa para } \\
\text { el grupo IS) }\end{array}$ & $\begin{array}{l}\text { El informe anecdótico de los } \\
\text { padres fue positivo con respecto } \\
\text { al progreso del niño después de } \\
\text { participar en el programa senso- } \\
\text { rialmente enriquecido. }\end{array}$ & $\begin{array}{l}\text { Estrategias de } \\
\text { base sensoriales } \\
\text { implementadas } \\
\text { dentro de un } \\
\text { programa grupal } \\
\text { de intervención } \\
\text { temprana }\end{array}$ \\
\hline $\begin{array}{l}\text { Bulkeley } \\
\text { et al., } \\
2016\end{array}$ & $\begin{array}{l}\text { TEA } \\
48-57 \mathrm{~m} .\end{array}$ & & $\begin{array}{l}\text { Destacó la importancia de edu- } \\
\text { car a los padres sobre las necesi- } \\
\text { dades del niño. }\end{array}$ & \\
\hline $\begin{array}{l}\text { Colyvas et } \\
\text { al. } 2010\end{array}$ & $\begin{array}{l}\text { Visitas de TO } \\
\text { en el hogar } \\
\text { Intervención } \\
\text { Temprana } \\
40 \text { videos } \\
\text { Edad no } \\
\text { reportada }\end{array}$ & & $\begin{array}{l}\text { La TO “basada en la participación' } \\
\text { fue significativamente más pro- } \\
\text { pensa a participar en la interac- } \\
\text { ción conjunta (apoya la interac- } \\
\text { ción cuidador-infante) que la TO } \\
\text { 'tradicional'. } \\
\text { El juego y el cuidado (principal- } \\
\text { mente en las comidas) fueron las } \\
\text { rutinas que predominantemente } \\
\text { utilizaron ambos grupos. }\end{array}$ & $\begin{array}{l}\text { TO 'tradicionales' } \\
\text { tenían más pro- } \\
\text { babilidades de } \\
\text { usar estrategias } \\
\text { de modelado }\end{array}$ \\
\hline
\end{tabular}




\begin{tabular}{|c|c|c|c|c|}
\hline $\begin{array}{l}\text { Temas } \\
\stackrel{\rightarrow}{\text { Autores }}\end{array}$ & Studio & Sensorial & Enfocado en el padre//familia & Grupal \\
\hline $\begin{array}{l}\text { Coulthard } \\
\text { et al. } 2017\end{array}$ & $\begin{array}{l}5 \text { semanas } \\
\text { de } \\
\text { intervención } \\
18 \mathrm{~m} .-4 \\
\text { años }\end{array}$ & $\begin{array}{l}\text { Grupos de juego sen- } \\
\text { sorial. Disfrutar juegos } \\
\text { que ensucian (con o } \\
\text { sin alimentos) se asocia } \\
\text { con mayor aceptación } \\
\text { de alimentos y el gusto } \\
\text { por probar fruta que el } \\
\text { grupo control. } \\
\text { No evaluó disfunciones } \\
\text { sensoriales }\end{array}$ & $\begin{array}{l}\text { El disfrute fue valorado por } \\
\text { padres e investigadores }\end{array}$ & \\
\hline $\begin{array}{l}\text { Gee \& } \\
\text { Nwora, } \\
2011\end{array}$ & $\begin{array}{l}\text { Grupo } \\
2-2.11 \text { años } \\
\text { y } \\
10 \text { cuida- } \\
\text { dores. } 8 \\
\text { sesiones }\end{array}$ & $\begin{array}{l}\text { Resultados positivos } \\
\text { estadísticamente signi- } \\
\text { ficativos fueron asocia- } \\
\text { dos al trabajo pesado, } \\
\text { vestibular y presión } \\
\text { profunda. Importancia } \\
\text { clínica (no estadística) } \\
\text { para las respuestas al } \\
\text { estímulo sensorial en } \\
\text { los niños y la eficacia } \\
\text { sensorial. }\end{array}$ & $\begin{array}{l}\text { La percepción de comprender } \\
\text { conceptos de procesamiento } \\
\text { sensorial aumentó al comparar } \\
\text { respuestas pre y post. }\end{array}$ & \\
\hline $\begin{array}{l}\text { Kingsley \& } \\
\text { Mailloux, } \\
2013\end{array}$ & $\begin{array}{l}\text { Revisión de } \\
18 \text { artículos } \\
0-5 \text { años }\end{array}$ & & $\begin{array}{l}5 \text { de } 18 \text { artículos abordaron inter- } \\
\text { venciones centradas en la familia } \\
\text { con evidencia moderada. } \\
6 \text { de los } 18 \text { estudios abordaron } \\
\text { la capacitación de los padres o la } \\
\text { relación padre / hijo. } \\
\text { Se necesitan más estudios para } \\
\text { determinar efectividad. } \\
\text { Los padres valoraron altamente } \\
\text { la capacitación que favorece sus } \\
\text { habilidades. } \\
\text { La eficacia y la satisfacción de los } \\
\text { padres aumentaron cuando las } \\
\text { intervenciones se integraron en } \\
\text { las rutinas y entornos familiares. }\end{array}$ & $\begin{array}{l}6 \text { de18 artículos } \\
\text { abordaron la } \\
\text { comunidad y el } \\
\text { entorno natural. } \\
\text { Los enfoques } \\
\text { combinados son } \\
\text { más efectivos. } \\
\text { Se necesita más } \\
\text { investigación } \\
\text { para determinar } \\
\text { el momento, la } \\
\text { frecuencia y la } \\
\text { duración de las } \\
\text { intervenciones }\end{array}$ \\
\hline
\end{tabular}




\begin{tabular}{|c|c|c|c|c|}
\hline $\begin{array}{c}\text { Temas } \\
\stackrel{\rightarrow}{\text { Autores }}\end{array}$ & Studio & Sensorial & Enfocado en el padre//familia & Grupal \\
\hline $\begin{array}{l}\text { Purpura et } \\
\text { al., } 2014\end{array}$ & $\begin{array}{l}\text { Hogar. } \\
20 \text { infantes } \\
\text { Síndrome de } \\
\text { Down. }\end{array}$ & $\begin{array}{l}\text { Estimulación tactil y } \\
\text { kinestésica de infantes. }\end{array}$ & $\begin{array}{l}\text { Masaje de padres a hijos para } \\
\text { facilitar el vínculo y relación } \\
\text { madre-hijo. } \\
\text { Mejoras en la maduración visual } \\
\text { a los } 6 \text { m. y desarrollo acelerado } \\
\text { hasta al menos } 12 \text { meses }\end{array}$ & \\
\hline $\begin{array}{l}\text { Spittle et } \\
\text { al., } 2015\end{array}$ & $\begin{array}{l}\text { Intervención } \\
\text { Temprana en } \\
\text { los primeros } \\
12 \mathrm{~m} \text {. }\end{array}$ & & $\begin{array}{l}\text { Las intervenciones incluyeron la } \\
\text { relación padre-hijo con un enfo- } \\
\text { que en el desarrollo infantil. } \\
25 \text { estudios respaldaron el } \\
\text { impacto positivo de la interven- } \\
\text { ción temprana en los primeros } 12 \\
\text { meses en el desempeño cogniti- } \\
\text { vo y motor. Poca evidencia para } \\
\text { efectos a largo plazo. }\end{array}$ & \\
\hline $\begin{array}{l}\text { Wainer et } \\
\text { al., } 2017\end{array}$ & $\begin{array}{l}\text { Revisión de } \\
\text { Intervención } \\
\text { Temprana en } \\
\text { TEA }\end{array}$ & & $\begin{array}{l}\text { Destaca la importancia de medir } \\
\text { los resultados de la intervención } \\
\text { a nivel familiar y de los padres. } \\
\text { Las medidas más comunes } \\
\text { incluyeron: crianza de los hijos, } \\
\text { autoeficacia, estrés, salud mental, } \\
\text { relaciones entre padres e hijos, y } \\
\text { funcionamiento familiar. } \\
\text { Proporciona una lista de herra- } \\
\text { mientas de evaluación. }\end{array}$ & $\begin{array}{l}\text { La mayoría de las } \\
\text { investigaciones } \\
\text { se centran en los } \\
\text { logros del niño }\end{array}$ \\
\hline
\end{tabular}

Nota: La tabla 3 presenta un resumen de las intervenciones en niños menores de 3 años por artículo, indicando el grupo de estudio, intervenciones de tipo sensorial, enfocadas en la familia y otros tipos de intervención.

Las intervenciones mencionadas en la literatura con respecto a las estrategias sensoriales fueron: (a) el masaje infantil, que demostró mejoría en la maduración visual de niños con síndrome de Down (Púrpura, et al. 2014); (b) el uso del juego sensorial 'sucio', que mostró un mayor consumo y satisfacción al comer frutas (Coulthard, Williamson, Palfreyman y Lyttle, 2017); y (c) la educación de los padres sobre conceptos y estrategias sensoriales durante un programa de juego grupal (Gee y Nwora, 2011). El segundo subtema está relacionado con la educación de los padres, la cual se considera central en la intervención con niños pequeños. A pesar de ello, algunos autores argumentan que esta educación a los padres no se está dando con la frecuencia que debería darse (Colyvas, et al., 2010). La literatura resalta el alto valor que los padres adjudican a la capacitación, a partir de la cual ellos mejoran sus habilidades para apoyar a sus hijos y sus familias (Bulkeley et al., 2016; Kingsley y Mailloux, 2013). La educación de los padres se ha correlacionado con una disminución de la ansiedad y la depresión de los padres, que se ve reflejada en efectos positivos en el desarrollo del niño 
tanto a corto plazo y hasta 24 meses más tarde (Benzies, Magill-Evans, Hayden y Ballantyne, 2013). Además, la eficacia y la satisfacción de los padres aumentó cuando las intervenciones se integraron dentro de las rutinas y los entornos familiares (Colyvas, et al., 2010).

Las intervenciones grupales que incorporaron tanto estrategias sensoriales como entornos sensorialmente enriquecidos mostraron mejores puntajes en las escalas cognitiva y de lenguaje en el Bayley 3, en niños con retraso en el desarrollo. Los niños con trastornos del procesamiento sensorial mostraron, además, mejores puntajes en la escala de motricidad gruesa en el mismo test (Blanche, Chang, Gutiérrez y Gunter, 2016). Igualmente, se encontraron resultados positivos asociados con intervenciones que incluyen trabajo pesado, estimulación vestibular y presión profunda en el programa de juego grupal (Gee y Nwora, 2011).

En general, en términos de intervención, esta revisión destaca: 1. La importancia de la intervención temprana, confirmando los resultados de otros estudios, como el de la revisión sistemática de Spittle, Orton, Anderson, Boyd, y Doyle (2015) quienes, en una revisión de 25 estudios, mostraron resultados positivos a nivel cognitivo y motor dentro de los primeros 12 meses de edad. 2. El uso combinado de enfoques de intervención, siendo más efectivo que las intervenciones que utilizaban sólo un enfoque. Sin embargo, se necesita más investigación para determinar el momento, la frecuencia y la duración de las intervenciones (Kingsley y Mailloux, 2013). 3. La inclusión de los padres en la medición de los resultados de la intervención temprana, sin embargo, se reporta que es poco frecuente (Kingsley y Mailloux, 2013; Benzies, et al., 2013; Wainer, et al., 2017).

\section{Discusión}

La presente revisión de la literatura da evidencia de patrones de disfunción en el procesamiento sensorial en niños menores de 3 años que varían dependiendo de la población o el diagnóstico (TEA, prematuro, retraso en el desarrollo, u otro). Los tipos de disfunción sensorial más comúnmente identificados en esta revisión (hipo/hiper-respuesta) se encuentran dentro de los 'desórdenes de regulación' identificados por Blanche y Gunter (2020), y no hay mención a problemas de tipo sensoriomotor, muy posiblemente debido a que el tipo de instrumentos utilizados miden primordialmente problemas de regulación. Esta revisión, señala cómo estos patrones de disfunción en el procesamiento sensorial identificados impactan negativamente la evolución del desarrollo y su desempeño funcional, cobrando importancia entre más pequeño sea el niño y en la medida que existen otros factores de riesgo. Si bien se ha reconocido la importancia de la intervención temprana, esta revisión destaca que el uso de varios enfoques de intervención es más efectivo que el uso individualizado de enfoques. Esto permite hacer un abordaje de las necesidades del niño no segmentada por áreas de desarrollo, y contemplar también el impacto del entorno y la familia como factores importantes para un resultado efectivo de la intervención.

Es importante reconocer que ésta no fue una revisión exhaustiva de la literatura existente y que otras revisiones respecto al tema de procesamiento sensoriales e intervenciones en el niño menor de 3 años ha seguido aumentando en los últimos años. Una de estas es la revisión de evaluaciones sensoriales de infantes por Eeles, et al. (2013), un tema no encontrado en esta revisión, pero que amerita atención en la medida en que buscamos seleccionar evaluaciones que nos proporcionen información relevante para una intervención efectiva. Esta revisión identificó 3 evaluaciones que cumplían con los criterios de uso clínico, confiabilidad, validez, y respuesta, nombrando The Sensory Rating Scale (CaseSmith J, Butcher L, Reed D.,1998) y The Infant/Toddler Sensory Profile (Dunn, 2002) como cuestionarios para padres de niños de o a 3 años, y el Test of Sensory Function in Infants (DeGangi y Greenspan, 1989) como evaluación de observación para niños de 4 a 18 meses.

Otros estudios recientes siguen analizando los patrones y pruebas utilizadas para medir el procesamiento sensorial en el niño prematuro (de Paula Machado, de Castro Magalhães, de Oliveira, y Bouzada, 2019; Niutanen, Harra, Lan y Metsäranta, 202O) un tema que se ha mantenido como un área de interés en la literatura a través de los años. Los resultados de éstos nuevos estudios confirman que el niño prematuro presenta mayor riesgo de disfunción sensorial que el niño a término, y aunque de Paula Machado et al. (2019) identifican mayor reactividad en el sistema táctil y vestibular similar a lo encontrado en esta revisión, la revisión sistemática de Niutanen, et al. (2020) encuentran una mayor variabilidad de disfunción sensorial con incidencias del $28-87 \%$ 
en modulación sensorial, un 9-70\% en procesamiento sensorial, y un $20-70 \%$ en procesamiento motor de base sensorial.

Entre otros estudios que hablan del impacto funcional que tiene la disfunción en procesamiento sensorial, están por ejemplo el estudio en niños de alto riesgo (Flanagan, Schoen, y Miller 2019) y el estudio en niños con autismo y retraso en el desarrollo (Kirby, et al., 2019). Flanagan et al. (2019) reportaron puntajes bajos en las escalas cognitiva y de lenguaje en el Bayley 3, mayor frecuencia de posiciones atípicas en la prueba Toddler and Infant Motor Evaluation, y menos búsqueda de sensaciones en el Toddler Sensory Profile 2 en los niños de alto riesgo de déficits en el procesamiento sensorial comparado con aquellos de bajo riesgo. Los autores sugieren que estos déficits a nivel funcional pueden estar asociados a los problemas en el procesamiento sensorial. El estudio de Kriby et al. (2019), confirma el impacto que imponen los patrones de disfunción sensorial sobre los niveles de estrés en la familia y de participación en actividades diarias.

Nuevos estudios se han enfocado en el procesamiento sensorial de niños menores con otros diagnósticos tales como: el síndrome de alcoholismo fetal (Fjeldsted y Xue, 2019), con alteraciones en su puntaje en procesamiento auditivo, bajo registro, sensibilidad sensorial y evasión sensorial según el ITSP; el síndrome $\mathrm{X}$ frágil (Baranek, Chin, Hess, Yankee, Hatton, y Hooper, 2002), donde experiencias de evasión sensorial internamente controladas se asocian a bajos niveles de participación escolar, autocuidado y juego, mientras que evasión al tacto externamente controlado se asocia a mayor independencia en autocuidado; y parálisis cerebral (Chorna, et al., 2015), donde un mejor procesamiento sensorial puede contribuir al progreso en la intervención motora.

Otras áreas de estudio del procesamiento sensorial que han surgido en la literatura incluyen: los cambios en el procesamiento sensorial en niños que han recibido anestesia (Berghmans, et al. 2018); la respuesta cerebral a la información tactil (Miguel, Gonçalves y Sampaio, 2020) y la alimentación (Blanche y Gunter 2020; Momin, et al., 2018). Dentro de los estudios recientes, se continúa haciendo énfasis en los servicios centrados en la familia. Por ejemplo, en un estudio cualitativo por Friberg (2018), los terapeutas ocupacionales comparten las estrategias que están utilizando con las familias para abordar las necesidades sensoriales y desarrollar dietas sensoriales, expandiendo sobre estrategias discutidas en esta revisión relacionadas a masaje infantil, juego sucio para promover interacción con alimentos, trabajo pesado o actividades sensoriales a nivel grupal y la preparación a los padres sobre las necesidades sensoriales de sus hijos.

Dentro de las limitaciones de esta revisión se encuentra el número limitado de artículos incluidos así como la no inclusión de mediciones utilizadas por cada estudio dentro del análisis, aunque se observa una tendencia a usar el perfil sensorial (Dunn, 2002) y el Sensory Processing Assessment for Young Children (SPA) (Baranek, 1999), diseñados para medir respuestas sensoriales de tipo hiper-hipo-respuesta, influenciando por lo tanto el tipo de patrones observados en el análisis. Igualmente, la revisión no incluye literatura en español que nos permita analizar diferencias en el procesamiento sensorial e intervenciones en los niños en países de habla hispana.

\section{CONCLUSIÓN}

La presente revisión proporciona una evidencia inicial de los patrones de procesamiento sensoriales que pueden presentar los niños menores de 3 años de edad y su impacto funcional, informando la evaluación y la intervención de terapistas ocupacionales que atienden este grupo de población, teniendo en cuenta la individualidad del niño, y la influencia de otros factores tales como el contexto familiar, cultural y social, y la salud del niño que puedan afectar su respuesta al estímulo sensorial y su desempeño. La revisión documenta intervenciones identificadas en la literatura donde se reconoce la importancia de la familia como uno de los contextos importantes para el desarrollo de estrategias de intervención (masajes, juego sensorial, trabajo pesado, entre otros), y destaca la necesidad de incluir evaluaciones para medir la satisfacción de los padres de familia en esta población menor de edad, ya que puede ser un indicativo de una intervención efectiva. Así mismo, se reconocen las limitaciones de esta revisión y el crecimiento de la literatura en estos temas a través de los años que amerita una mirada más profunda, que pueda contribuir a guiar la práctica profesional en la intervención temprana dentro del contexto latinoamericano. 


\section{RefERENCIAS BiBLIOGRÁFICA}

Arbesman, M., Lieberman, D. \& Berlanstein, D. R. (2013). Method for the systematic reviews on Occupational Therapy and early intervention and early childhood services. American Journal of Occupational Therapy, 67, 389-394. http://dx.doi.org/10.5014/ajot.2013.007039

Ayres, A. J. (2005). Sensory integration and the child. Los Angeles: Western Psychological Services.

Baranek, G. T. (1999). Sensory Processing Assessment for Young Children (SPA). Unpublished manuscript, University of North Carolina at Chapel Hill.

Baranek, G. T., Boyd, B. A., Poe, M. D., David, F. J., \& Watson, L. R. (2007). Hyperresponsive sensory patterns in young children with autism, developmental delay, and typical development. American Journal of Mental Retardation, 11(4), 233-245.

Baranek, G. T., Chin, Y. H., Hess, L. M. G., Yankee, J. G., Hatton, D. D., \& Hooper, S. R. (2002). Sensory processing correlates of occupational performance in children with fragile X syndrome: Preliminary findings. American Journal of Occupational Therapy, 56, 538-546

Baranek, G. T., Watson, L. R., Boyd, B. A., Poe, M. D., David, F. J., \& McGuire, L. (2013). Hyporesponsiveness to social and nonsocial sensory stimuli in children with autism, children with developmental delays, and typically developing children. Development and Psychopathology, 25, 307-320.

Ben-Sasson, A., Cermak, S. A., Orsmond, G. I., Tager-Flusberg, H., Carter, A. S., Kadlec, M. B., \& Dunn, W. (2007). Extreme sensory modulation behaviors in toddlers with autism spectrum disorders. American Journal of Occupational Therapy, 61, 584-592.

Benzies, K. M., Magill-Evans, J. E., Hayden, K. A., \& Ballantyne, M. (2013). Key components of early intervention programs for preterm infants and their parents: A systematic review and meta-analysis. $B M C$ Pregnancy \& Childbirth, 13(Suppl 1), S10.

Berghmans, J. M., Poley, M. J., van der Ende, J., Rietman, A., Glazemakers, I., Himpe, D., Verhulst, F. C., \& Utens, E. (2018). Changes in sensory processing after anesthesia in toddlers. Minerva anestesiologica, 84(8), 919-928. https://doi.org/10.23736/S0375-9393.18.12132-8

Blanche, E.I., Gunter, J.S., 2020. Sensory Processing Disorders. In: Benson, J.B. (Ed.), Encyclopedia of Infant and Early Childhood Development, 2nd edition. vol. 3, Elsevier, 116-124. https://dx.doi.org/10.1016/ B978-0-12-809324-5.23602-X ISBN: 9780128165126

Blanche, E. I., Chang, M. C., Gutiérrez, J., \& Gunter, J. S. (2016). Effectiveness of a sensory-enriched early intervention group program for children with developmental disabilities. American Journal of Occupational Therapy, 70(5), 7005220010p1-7005220010p8.

Bröring, T., Oostrom, K. J., Lafeber, H. N., Jansma, E. P., \& Oosterkaan, J. (2017). Sensory modulation in preterm children: Theoretical perspective and systematic review. PLoS ONE, 12(2), e0170828p1- e0170828p23. https://doi.org/10.1371/journal.pone.0170828
Bulkeley, K., Bundy, A., Roberts, J., \& Einfeld, S. (2016). Family-centered management of sensory challenges of children with autism: Single-case experimental design. American Journal of Occupational Therapy, 70(5), 7005220040p1-7005220040p8.

Case-Smith, J. (2013). Systematic review of interventions to promote social-emotional development in young children with or at risk for disability. American Journal of Occupational Therapy, 67, 395-404. http://dx.doi.org/10.5014/ajot.2013.004713

Case-Smith J, Butcher L, Reed D. (1998). Parents' report of sensory responsiveness and temperament in preterm infants. American Journal of Occupational Therapy, 52, 547-55.

Case-Smith, J., Frolek Clark, G. J., \& Schlabach, T. L. (2013). Systematic review of interventions used in occupational therapy to promote motor performance for children ages birth-5 years. American Journal of Occupational Therapy, 67, 413-424. http://dx.doi.org/10.5014/ ajot.2013.005959

Clark, G. F., \& Kingsley, K. L. (2020). Occupational Therapy Practice Guidelines for Early Childhood: Birth-5 Years. American Journal of Occupational Therapy, 74(3), 7403397010p1-7403397010p42. https:// doi.org/10.5014/ajot.2020.743001

Colyvas, J. L., Sawyer, L. B., \& Campbell, P. H. (2010). Identifying strategies early intervention occupational therapists use to teach caregivers. American Journal of Occupational Therapy, 64, 776-785.

Chorna, O., Solomon, J. E., Slaughter, J. C., Stark, A. R., \& Maitre, N. L. (2014). Abnormal sensory reactivity in preterm infants during the first year correlates with adverse neurodevelopmental outcomes at 2 years of age. Arch Dis Child Fetal Neonatal Ed 99: F475-F479. http://dx.doi.org/10.1136/archdischild-2014-306486.

Chorna, O., Heathcock, J., Key, A., Noritz, G., Carey, H., Hamm, E., ... Maitre, N. L. (2015). Early childhood constraint therapy for sensory/ motor impairment in cerebral palsy: a randomised clinical trial protocol. BMJ open, 5(12), e010212p1- e010212p13.

Coulthard, H., Williamson, I., Palfreyman, Z. \& Lyttle, S. (2017). Evaluation of a pilot sensory play intervention to increase fruit acceptance in preschool children. Appetite 120, 609-615.

DeGangi, G. A.\& Stanley I. Greenspan, S. I. (1989). Test of Sensory Functions in Infants. Los Angeles: Western Psychological Services.

DeMaster, D., Bick, J., Johnson, U., Montroy, J. J., Landry, S., \& Duncan, A. F. (2019). Nurturing the preterm infant brain: leveraging neuroplasticity to improve neurobehavioral. Pediatric Research, 85, 166-175. de Paula Machado, A., de Castro Magalhães, L., de Oliveira, S. R., \& Bouzada, M. (2019). Is sensory processing associated with prematurity, motor and cognitive development at 12 months of age. Early human development, 139, 104852p1-104852p6. https://doi.org/10.1016/j. earlhumdev.2019.104852

DeSantis, A., Harkins, D., Tronick, E., Kaplan, E., \& Beeghly, M. (2011). Exploring an integrative model of infant behavior: What is the relationship among temperament, sensory processing, and neurobehavioral measures? Infant Behavior and Development, 34, 280-292. 
Dunn, W. (2002). Infant toddler sensory profile manual. New York: Psychological Corporation.

Eeles, A. L., Spittle, A. J., Anderson, P. J., Brown, N., Lee, K. J., Boyd, R. N., \& Doyle, L. W. (2013). Assessments of sensory processing in infants: a systematic review. Developmental Medicine \& Child Neurology, 55(4), 314-326.

Fjeldsted, B., \& Xue, L. (2019). Sensory Processing in Young Children with Fetal Alcohol Spectrum Disorder. Physical \& occupational therapy in pediatrics, 39(5), 553-565. https://doi.org/10.1080/019 42638.2019.1573775

Flanagan, J. E., Schoen, S. \& Miller, L. J. (2019). Early Identification of Sensory Processing Difficulties in High-Risk Infants. American Journal of Occupational Therapy, 73 (2), 7302205130p1-7302205130p9. https:// doi.org/10.5014/ajot.2018.028449

Frolek Clark, G. J., \& Schlabach, T. L. (2013). Systematic review of occupational therapy interventions to improve cognitive development in children ages birth-5 years. American Journal of Occupational Therapy, 67, 425-430. http://dx.doi.org/10.5014/ajot.2013.006163

Friberg, D. (2018). Understanding the Use of Family-Centered Principles by Early Intervention Occupational Therapists in the Development of Sensory Diets. American Journal of Occupational Therapy, 72, (4_Supplement_1) 7211505153p1-7211505153p1-. https://doi. org/10.5014/ajot.2018.72S1-PO7017

Gee, B. M., \& Nwora, A. J. (2011). Enhancing caregiver perceptions using center-based sensory processing playgroups: Understanding and efficacy. Journal of Occupational Therapy, Schools, \& Early Intervention, 4, 276-290.

Germani, T., Zwaigenbaum, L., Bryson, S., Brian, J., Smith, I., Roberts, W., Szatmari, P,... Vaillancourt, T.(2014). Brief Report: Assessment of Early Sensory Processing in Infants at High-Risk of Autism Spectrum Disorder. Journal Autism Developmental Disorder, 44, 3264-3270. DOI 10.1007/s10803-014-2175-x

Gutiérrez, J., Chang, M. \& Blanche, E. (2016). Sensory Processing In Toddlers Diagnosed With Autism Spectrum Disorder (ASD). Revista Chilena de Terapia Ocupacional, 16(1), 89 - 98. ISSN 0717-6767

Howe, T.-H., \& Wang, T.-N. (2013). Systematic review of interventions used in or relevant to occupational therapy for children with feeding difficulties ages birth-5 years. American Journal of Occupational Therapy, 67, 405-412. http://dx.doi.org/10.5014/ajot.2013.004564

Kingsley, K. \& Mailloux, Z. (2013). Evidence for the effectiveness of different service delivery models in early intervention services. American Journal of Occupational Therapy, 67, 431-436. http://dx.doi. org/10.5014/ajot.2013.006171

Kirby, A. V., Williams, K. L., Watson, L. R., Sideris, J., Bulluck, J., \& Baranek, G. T. (2019). Sensory features and family functioning in families of children with autism and developmental disabilities: Longitudinal associations. American Journal of Occupational Therapy, 73(2), 7302205040p1-7302205040p14. https://doi.org/10.5014/ ajot.2018.027391
Koch, K. R., \& Moore, B. M. (2015). Relating neurodevelopment to early intervention special education: Implications for developing best practices. International Journal of Early Childhood Special Education, 7(1), 51-68.

Lane, S. J., \& Schaaf, R. C. (2010). Examining the neuroscience evidence for sensory-driven neuroplasticity: Implications for sensory-based occupational therapy for children and adolescents. American Journal of Occupational Therapy, 64, 375-390. doi: 10.5014/ajot.2010.09069

Miguel, H. O., Gonçalves, Ó. F. \& Sampaio, A. (2020). Behavioral response to tactile stimuli relates to brain response to affective touch in 12-month-old infants. Developmental psychobiology, 62(1), 107-115. https://doi.org/10.1002/dev.21891

Mitchell, A. W., Moore, E. M., Roberts, E. J., Hachtel, K. W., \& Brown, M. S. (2015). Sensory processing disorder in children ages birth-3 years born prematurely: A systematic review. American Journal of Occupational Therapy, 69(1), 6901220030p1-6901220030p11. http:// dx.doi.org/10.5014/ajot.2015.013755.

Mulligan, S., \& White, B. P. (2012). Sensory and motor behaviors of infant siblings of children with and without autism. American Journal of Occupational Therapy, 66, 556-566.

Momin, S. R., Hughes, S. O., Elias, C., Papaioannou, M. A., Phan, M., Vides, D., \& Wood, A. C. (2018). Observations of Toddlers' sensory-based exploratory behaviors with a novel food. Appetite, 131, 108-116. https://doi.org/10.1016/j.appet.2018.08.035

Mundkur, N. (2005). Neuroplasticity in children. The Indian Journal of Pediatrics, 72, 855-857 https://doi.org/10.1007/BF02731115

Niutanen, U., Harra, T., Lano, A., \& Metsäranta, M. (2020). Systematic review of sensory processing in preterm children reveals abnormal sensory modulation, somatosensory processing and sensory-based motor processing. Acta Paediatrica, 109(1), 45-55.

Park, H. Y., Maitra, K., Achon, J., Loyola, E., \& Rincón, M. (2014). Effects of early intervention on mental or neuromusculoskeletal and movementrelated functions in children born low birthweight or preterm: A metaanalysis. American Journal of Occupational Therapy, 68, 268-276. http://dx.doi.org/10.5014/ajot.2014.010371

Philpott-Robinson, K., Lane, A. E., \& Harpster, K. (2016). Sensory Features of toddlers at risk for autism spectrum disorder. American Journal of Occupational Therapy, 70(4), 7004220010p1-7004220010p8.

Púrpura, G., Tinelli, F., Bargagna, S., Bozza, M., Bastiani, L., \& Cioni, G. (2014). Effect of early multisensory massage intervention on visual functions in infants with Down syndrome. Early Human Development, 90, 809-813.

Smith, I., Roberts, W., Szatmari, P., Roncadin, C., Sacrey, L. A. R., Garon, N., \& Vaillancourt, T. (2014). Brief report: Assessment of early sensory processing in infants at high-risk of autism spectrum disorder. Journal of Autism and Developmental Disorders, 44, 3264-3270.

Spittle, A., Orton, J., Anderson, P. J., Boyd, R., \& Doyle, L. W. (2015). Early developmental intervention programs provided post hospital discharge to prevent motor and cognitive impairment in preterm infants. Cochrane Database of Systematic Reviews, 11, 1-84. 
Tauman, R., Avni, H., Drori-Asayag, A., Nehama, H., Greenfeld, M., \& Leitner, Y. (2017). Sensory profile in infants and toddlers with behavioral insomnia and/or feeding disorders. Sleep Medicine, 32, 83-86.

Vasak, M., Williamson, J., Garden, J., \& Zwicker, J. G. (2015). Sensory processing and sleep in typically developing infants and toddlers. American Journal of Occupational Therapy, 69(4), 6904220040p1-6904220040p8. http://dx.doi.org/10.5014/ajot.2015.015891

Wainer, A. L., Hepburn, S., \& McMahon Griffith, E. (2017). Remembering parents in parent-mediated early intervention: An approach to examining impact on parents and families. Autism 21(1) 5-17. DOI: $10.1177 / 1362361315622411$

Weiss, S. J. \& Wilson, P. (2006). Origins of tactile vulnerability in highrisk infants. Advances in Neonatal Care, 6(1): 25-36. 